Check for updates

Cite this: New J. Chem., 2017, 41,4145

Received 16th January 2017. Accepted 12th April 2017

DOI: 10.1039/c7nj00187h

rsc.li/njc

\section{Pd nanocube decoration onto flexible nanofibrous mats of core-shell polymer-ZnO nanofibers for visible light photocatalysis $\dagger$}

\author{
Osman Arslan, (D) *a Fuat Topuz, ${ }^{a}$ Hamit Eren, ${ }^{b}$ Necmi Biyikli ${ }^{c}$ and Tamer Uyar (D) *ab
}

\section{Introduction}

The transition and efficient production from UV dependent to visible light photocatalysis requires fine engineering of the nanostructures concerning the nanoparticle size, surface, defect states, band gap energy and shape. ${ }^{1-3}$ It is remarkably important to fabricate visible light active nanostructures which can modulate the visible light to obtain nanostructures to be used in remediation, environmental purification, photovoltaics and $\mathrm{H}_{2}$ production. ${ }^{4-8}$ UV dependent photocatalysis is widely known and has been investigated in recent years $;^{2}$ moreover, on the basis of semiconductors and nanomaterials, visible light active structures can be obtained by doping, QD sensitization,

\footnotetext{
${ }^{a}$ UNAM-National Nanotechnology Research Center, Bilkent University, 06800, Ankara, Turkey.E-mail: arslan@unam.bilkent.edu.tr, uyar@unam.bilkent.edu.tr

${ }^{b}$ Institute of Materials Science \& Nanotechnology, Bilkent University, Ankara, 06800, Turkey

${ }^{c}$ Electrical and Computer Engineering Department, Utah State University, Logan, UT 84322-4120, USA

$\dagger$ Electronic supplementary information (ESI) available: Fiber diameter distribution of the PAN-NF and ZnO@PAN-NF, EDX analysis of PAN-NF and ZnO@PAN-NF, size distributions of the Pd nanocubes, enlarged SEM images and SAED pattern of ZnO@PAN-NF, XRD of Pd@ZnO@PAN-NF samples, visual observation of the Pd nanocube decoration onto the ZnO@PAN-NF, SEM and EDX mapping of Pd@ZnO@PAN-NF samples, UV-vis absorption spectra of ZnO@PAN-NF and Pd@ZnO@PAN-NF, MB decomposition by ZnO@PAN-NF and Pd@ZnO@PAN-NF samples, XPS and ICP-MS analyses of the Pd@ZnO@PAN-NF samples are given and available. See DOI: 10.1039/c7nj00187h
}

metal nanoparticle coupling (plasmonic enhancement), defect engineering or some other special methods to yield better visible range electron-hole separation..$^{9-11}$

Highly effective visible light active studies can be given as inspiring examples, such as magnetically recovered visible light active structures, ${ }^{12}$ graphene and $\mathrm{Ag}$ modified $2 \mathrm{D} \mathrm{Bi}_{2} \mathrm{WO}_{6}$ nanosheets for visible light photocatalytic activity applications, ${ }^{13}$ enhanced charge carrier separation and photocatalytic activity with common $\left(\mathrm{TiO}_{2}, \mathrm{WO}_{3}\right.$ and $\left.\mathrm{ZnO}\right)$ nanostructures, ${ }^{14}$ non-metal doped enhanced photocatalytic activity of the $\mathrm{ZnO}$ structures, ${ }^{15}$ UV cured polymeric and photocatalytically active nanoparticles, ${ }^{16}$ biomineralized magnetic particles coated with chitosan and metal ions for catalytic applications. ${ }^{17}$ These nanostructures are generally a combination of the metal oxide and metallic nanostructures in which the interfacial interaction was designed with a special route to enhance the plasmonic effect. Additionally, doping effects with non-metallic and metallic entities were also investigated from an increased catalytic effect perspective. From this viewpoint, a direct semiconductor $\mathrm{ZnO}$ can be used which has wide band gap $(3.37 \mathrm{eV})$ and strong exciton binding energy ( $60 \mathrm{meV})$, providing a great deal of interest for sunscreens, ${ }^{18}$ antimicrobial agents, ${ }^{19}$ solar energy conversion, ${ }^{20}$ photocatalysis and environmental remediation. ${ }^{21-23}$ In the electron/hole separation concept, UV light absorption causes electron/hole pairs and charge separation due to the large surface to volume ratios and the photon confinement effect, and exciton transfer gains a special importance for an improved photovoltaic effect. An effective way to improve the band absorption/emission 
features of $\mathrm{ZnO}$ is localized surface plasmon resonance (SPR) mediated enhancement. ${ }^{24,25} \mathrm{SPR}$ is a collective oscillation of the electromagnetic field of incident light in the visible and IR regions. When a $\mathrm{ZnO}$ defect features and SPR is matched, the surface plasmons (SP) of the metallic NPs can be excited via the defect emission energy of $\mathrm{ZnO}$ nanomaterials. ${ }^{26,27}$ It is well known that if the energy of defect emission and SPR matches, the surface plasmons (SP) of metallic and suitable NPs can be excited via the defect emission energy of $\mathrm{ZnO}$ nanomaterials. Theoretically and practically, these excited surface plasmons of metal NPs can be transferred into the $\mathrm{CB}$ of $\mathrm{ZnO}$ nanomaterials. $^{28,29}$ During this process, due to the reasons which do not allow the electrons to be transferred, the rest of the electrons can recombine with electron hole pairs in the valence band VB. Therefore, when metal nanoparticles are decorated onto the $\mathrm{ZnO}$, two possible effects can be observed which are the inducement of the free electrons to flock and the establishment of a platform for free electron transfer to the interface. The obtained free electrons can shift or pin the Fermi level of this nanocomposite structure and increase the efficiency of the photovoltaic features and devices by about $10-15 \%$ and also enhance the photocatalytic activity toward organics decomposition and decomposing water. These nanomaterials contribution to the enhanced separation of the excitons is huge but their synthesis generally involve many and aggressive steps and cleaning procedures.

In this study, we have engineered a metal oxide $(\mathrm{ZnO}) /$ metal (Pd) nanocube interface and enhanced the performance of the photovoltaic character arising from controlled defects of the ZnO nanolayers. Firstly, core-shell nanofibers were fabricated by coating the electrospun PAN nanofibers with $\sim 40 \mathrm{~nm}$ of a $\mathrm{ZnO}$ layer by an atomic layer deposition (ALD) method (ZnO@PAN-NF). Moreover, deposition of the cubic Pd nanoparticles with different sizes varying between 7, 13 and $22 \mathrm{~nm}$ was performed with a controlled immersion/curing technique (Fig. 1).

Once the cubic Pd nanoparticles had attached to the surface of the ZnO layer, the Pd@ZnO@PAN-NF nanofibrous mat showed a higher efficiency for visible light photocatalysis due to the good contact and increased charge transfer. The enhanced visible light photocatalytic activity of Pd@ZnO@PAN-NF was studied and the relative improvement was established. Our results were convincing for portraying the presented method as one of a promising architectural design for the future of defect enhanced mediated visible light electron/hole separation and plasmonic applications.

\section{Experimental}

\subsection{Materials}

Polyacrylonitrile (PAN) $\left(M_{\mathrm{w}}: 150000\right)$ was purchased from Scientific Polymer Products, Inc, USA, dimethylformamide (DMF, $\geq 99 \%$ Riedel Hahn) was used as solvent. ALD of the $\mathrm{ZnO}$ was performed using diethylzinc (DEZn, Sigma-Aldrich) and HPLC grade water $\left(\mathrm{H}_{2} \mathrm{O}\right)$ as the precursor and oxidant, respectively. Sodium tetrachloropalladate(II) hydrate $\left(\mathrm{Na}_{2} \mathrm{PdCl}_{4} \cdot x \mathrm{H}_{2} \mathrm{O}, \quad 99.95 \%\right.$, Alfa Aesar), polyvinylpyrrolidone (PVP, $M_{\mathrm{w}}: 1300000$, Aldrich)

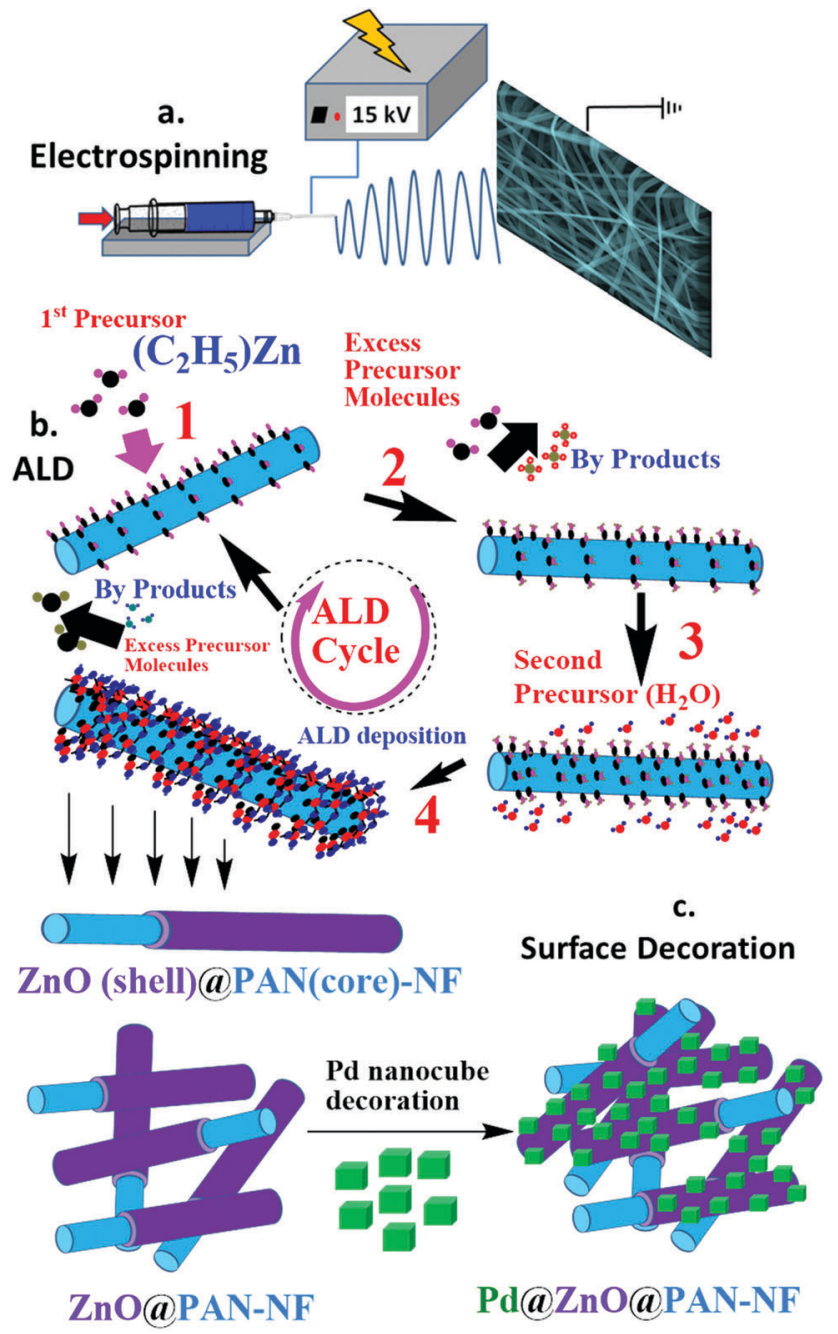

Fig. 1 Schematic representations of (a) electrospinning of PAN nanofibers, (b) ALD coating of ZnO nanolayer onto PAN nanofiber, and (c) Pd nanocube surface decoration on ZnO@PAN-NF.

potassium bromide (KBr, 99\%, Sigma-Aldrich) and ascorbic acid (AA, Merck 99\%) were used for the palladium nanocube synthesis. Methylene blue ( $\mathrm{MB}, \mathrm{C}_{16} \mathrm{H}_{18} \mathrm{~N}_{3} \mathrm{ClS}$,) was purchased from Sigma-Aldrich (Germany). The stock solution of $\mathrm{MB}$ was prepared and the concentration of $\mathrm{MB}$ in each aqueous solution was measured on an UV-vis spectrophotometer while taking the absorption at $660 \mathrm{~nm}$.

\subsection{Electrospinning of PAN nanofibers (PAN-NF)}

For the fabrication of PAN-NF, $12 \%(\mathrm{w} / \mathrm{v})$ PAN was dissolved in DMF and then this polymer solution was used for the electrospinning. The concentration and other parameters were optimized as previously for the electrospinning of bead-free and uniform PAN nanofibers. $15 \mathrm{kV}$ was applied (AU Series, Matsusada) between the syringe needle and metal collector and the flow rate of the PAN solution was $1 \mathrm{ml} \mathrm{h}^{-1}$. Electrospun nanofibers were collected on clean aluminum foil which was attached onto the metal collector located at $10 \mathrm{~cm}$ from the syringe tip. The chamber temperature was $25{ }^{\circ} \mathrm{C}$ and a $22 \%$ 
relative humidity was observed during the electrospinning process. There was no additional treatment after the electrospinning process and nanofibers were analyzed analytically to investigate their chemical and physical properties.

\subsection{Atomic layer deposition (ALD) of $\mathrm{ZnO}$ on PAN nanofibers (ZnO@PAN-NF)}

For the ALD of the $\mathrm{ZnO}$ layer on the electrospun PAN nanofibers, an air sensitive $\mathrm{Zn}\left(\mathrm{C}_{2} \mathrm{H}_{5}\right)_{2}$ precursor was used. $\mathrm{ZnO}$ deposition was carried out at $200{ }^{\circ} \mathrm{C}$ in a Savannah S100 ALD reactor (Cambridge Nanotech Inc.). $\mathrm{N}_{2}$ gas was used as a carrier for the deposition at a flow rate of $20 \mathrm{sccm} .400$ cycles were applied via an exposure mode (a trade-mark of Ultratech/Cambridge Nanotech Inc.) where dynamic to static vacuum switching was applied prior to pulses by the valve. After the cycles and predetermined exposure time, the vacuum was switched back to the dynamic mode for unreacted molecules and excessive by products. A full determination of one ALD cycle can be described as follows: valve $\mathrm{OFF} / \mathrm{N}_{2}$ flowset to $10 \mathrm{sccm} / \mathrm{H}_{2} \mathrm{O}$ pulse $(0.015 \mathrm{~s}) /$ exposure $(10 \mathrm{~s}) /$ valve $\mathrm{ON} / \mathrm{N}_{2}$ purge $(20 \mathrm{sccm}, 10 \mathrm{~s}) /$ valve $\mathrm{OFF} /$ $\mathrm{N}_{2}$ flow set to $10 \mathrm{sccm} / \mathrm{DEZn}$ pulse $(0.015 \mathrm{~s}) /$ exposure $(10 \mathrm{~s}) /$ valve $\mathrm{ON} / \mathrm{N}_{2}$ purge (20 sccm, $\left.10 \mathrm{~s}\right)$.

\subsection{Synthesis of anisotropic Pd nanocubes}

For the synthesis of Pd cubic nanoparticles, $0.21 \mathrm{~g}$ PVP, 1.2 (Pd-22), 0.30 (Pd-13), 0.15 (Pd-7) g, KBr and ascorbic acid mixture was prepared in $16 \mathrm{ml}$ water. The solution was stirred for about 30 minutes and then $0.114 \mathrm{~g} \mathrm{Na}_{2} \mathrm{PdCl}_{4} \cdot x \mathrm{H}_{2} \mathrm{O}(x \approx 3)$ in $5 \mathrm{ml} \mathrm{H}_{2} \mathrm{O}$ was added. Then the reaction was conducted at $80{ }^{\circ} \mathrm{C}$ during 3 hours. The available liquid part was removed by centrifuge at $1100 \mathrm{rpm}$ for 15 minutes. Water was removed by rotavapor and repetitive mixtures were prepared with toluene to remove the water completely. Nanoparticles were analyzed with different analytical techniques and they were redispersed in water for the deposition onto ZnO@PAN-NF.

\subsection{Deposition of Pd nanocubes onto $\mathrm{ZnO@PAN} \mathrm{nanofibers}$ (Pd@ZnO@PAN-NF)}

For the deposition of the Pd nanocubes with different sizes (7, 13 and $22 \mathrm{~nm}), 2 \times 2 \mathrm{~cm}$ ZnO@PAN nanofibrous mats were immersed into the aqueous Pd nanocube dispersions prepared prior to the deposition process. Immersed ZnO@PAN nanofibrous mats were stirred at $200 \mathrm{rpm}$ for 30 minutes and dried at $110{ }^{\circ} \mathrm{C}$ for 5 minutes. The Pd nanocube deposited nanofibrous mats (Pd@ZnO@PAN-NF) showed relatively darker colors. The analytical investigations were accordingly conducted together with the photocatalytical tests for these three Pd@ZnO@PAN-NF samples.

\subsection{Measurements and characterization}

X-ray photoelectron spectroscopy (XPS) spectra were obtained by a flood gun charge neutralizer system equipped with a monochromated Al $\mathrm{K} \alpha$ X-ray source $(h \nu=1486.6 \mathrm{eV})$ from a $400 \mathrm{~mm}$ spot size on the nanofibrous web. Wide energy survey scans were recorded in the $\mathbf{0}-1360 \mathrm{eV}$ binding energy range, at a detector pass energy of $200 \mathrm{eV}$ and with an energy step size of $1 \mathrm{eV}$. High resolution spectra were obtained at a pass energy of
$50 \mathrm{eV}$ and with energy steps of $0.1 \mathrm{eV}$ for each atom. The surface and elemental composition of the nanofibers was performed by XPS (Thermo Scientific). X-ray diffractometer (XRD) (X'Pert powder diffractometer, PANalytical) was used to determine the $\mathrm{X}$-ray diffraction pattern of the $\mathrm{ZnO}$ coated sample with $\mathrm{Cu} \mathrm{K} \alpha$ radiation in the $2 \theta=5-80^{\circ}$ range. The elemental composition investigations (EDX mapping) of the nanofibers were performed together with morphology and the diameter analyses with a scanning electron microscope (SEM) (Quanta 200 FEG, FEI). In order to avoid the electron charging effect, nanofibrous webs were coated with $5 \mathrm{~nm}$ Au (with PECS-682) prior to the SEM imaging. Average fiber diameters were calculated by counting and measuring 100 fibers and plotting them with respect to their frequencies. Non-linear fitting on the obtained fiber diameter gave an average value with a calculated statistical standard deviation. Additional morphological, surface, and diameter examinations were conducted by transmission electron microscopy (TEM, Tecnai G2 F30) equipped with an EDS. ZnO deposition and decoration of the cubic nanoparticles onto the nanofibers was studied by using STEM-EDX (scanning transmission electron microscopy-energy dispersive X-ray analysis) and high angle annular dark field-STEM (HAADF-STEM) images with atomic mapping have been obtained. The presence of Pd in the nanofibrous mats $\left(\sim 1 \times 1 \mathrm{~cm}^{2}\right.$ mat of the Pd@Zn@PAN-NF samples, $\sim 0.7 \mathrm{mg}$ each) was studied by Inductively Coupled Plasma-Mass Spectrometry (ICP-MS, Thermo, X Series II). For the detection of the Pd nanoparticles, nanofibers were dissolved in a $2 \mathrm{ml} \mathrm{HNO}_{3} / \mathrm{HCl}$ mixture. Decomposition of the MB was monitored by UV-vis Spectrophotometry (Cary 100). Photoluminescence properties and defect structure characteristics were detected by Fluorescence Spectrophotometer (Cary, Eclipse).

\subsection{Visible-light photocatalytic activity}

The visible range photocatalytic activities of the Pd@ZnO@ PAN-NF versus ZnO@PAN-NF were analyzed through the visible range photo induced degradation of $\mathrm{MB}$ dye in aqueous medium. $2 \times 2 \mathrm{~cm}$ mats were utilized into the MB dye solution and stored in darkness for $1 \mathrm{~h}$ to bring the $\mathrm{MB}$ dye into equilibrium. $2 \times 2 \mathrm{~cm}$ mats in this MB solution were then illuminated with a Newport 91192 solar simulator equipped with $300 \mathrm{~W}$ xenon arc. The distance between the source and solution was $15 \mathrm{~cm}$. Aliquots were taken from the dye solution to detect the concentration variations in the MB solutions and measured using a UV-vis spectrophotometer (Varian Cary 100) with defined time intervals. The decomposition rate of MB degradation was quantified via a first order exponential fit $\left(y=y_{0}+A^{e-x / t}\right)$ for the obtained aliquots and data set.

\section{Results and discussion}

Electrospinning is a very promising technique for the fabrication of diverse functional nanofibers ${ }^{30-32}$ as portrayed in Fig. 1a. In our previous study, we have already optimized the electrospinning conditions for PAN in order to obtain bead-free and uniform nanofibers. $^{33,34}$ The SEM imaging of the electrospun PAN 
nanofibers confirmed the bead-free and uniform morphology of the sample (Fig. 2a). The average fiber diameter (540 $\pm 30 \mathrm{~nm})$ and distribution of PAN-NF can be found in Fig. S1 (ESI $\dagger$ ) together with the ALD of the $\mathrm{ZnO}$ coated (600 $\pm 30 \mathrm{~nm}$ ) sample (ZnO@PAN-NF) (Fig. S1, ESI $\dagger$ ). SEM imaging provided useful information concerning the PAN-NF (Fig. 2a) and ZnO@PAN-NF (Fig. 2b) samples. From the SEM data, the surface character and morphological features and from the EDX analysis (Fig. 2c) the atomic compositions were obtained. Electrospun PAN nanofibers were subjected to ALD cycles for the deposition of the $\mathrm{ZnO}$ nanolayer (Fig. 1b) in order to obtain the core-shell ZnO@PAN-NF. The SEM images (Fig. 2b) after ALD of the ZnO showed that the fiber structure of the PAN was preserved for ZnO@PAN-NF and there was no significant morphological change except a slight variation due to the $\mathrm{ZnO}$ grainy structure. Temperature dependency is an important parameter for the ALD since thermal stability of the polymeric nanofiber template plays an important role for the formation of $\mathrm{ZnO}$ and compatibility with the ALD parameters. Since a relatively low temperature $\left(200{ }^{\circ} \mathrm{C}\right)$ is applicable for the ALD of $\mathrm{ZnO}$, organic structures can be preserved without thermal decomposition of the polymeric nanofiber matrix. Investigations also showed that even though the general fiber morphology showed no change, the surface roughness increased after the ALD process due to the grainy and crystalline structure of $\mathrm{ZnO}$. The increase in average fiber diameter of PAN-NF from $540 \pm 30 \mathrm{~nm}$ to $600 \pm 30 \mathrm{~nm}$ for ZnO@PAN-NF was due to the additional nanocoating $(\sim 40 \mathrm{~nm})$ of $\mathrm{ZnO}$ by the ALD process (Fig. 1b and Fig. S1, ESI $\dagger$ ). A relatively rough surface after the ALD of the ZnO is highly desired as it may increase the visible light photocatalytic efficiency after Pd nanocube coupling due to the plasmonic back feeding of the oxygen defects. EDX quantitative measurement (Fig. S2, ESI $\dagger$ ) and atomic mapping (Fig. 2c) were employed to show the homogeneity of the $\mathrm{ZnO}$ layer on the PAN nanofibers which supports the general observations (Fig. 2c). ZnO nanolayers perfectly cover the PAN fibers, providing a defect abundant photocatalytic activation platform for the UV absorbed photons and plasmonic transferred electrons. Previously, we have demonstrated that flexible polymericinorganic core-shell nanofibers can be fabricated by ALD of well crystallized $\mathrm{ZnO}$ nanolayers on the electrospun polymeric nanofibers for photocatalysis. ${ }^{33}$ The increase in photocatalytic activity depends on the light absorption with surface defects which generally form as shallow or deep sub-band gap states. Very promisingly, the ALD of $\mathrm{ZnO}$ nanolayers on the PAN electrospun nanofibers resulted in an enhanced UV light photocatalytic activity. Since defects are the result of oxygen vacancies creating interbands but also act as self-dopants inducing a band gap energy, this effort can go one step further by plasmonic contribution into the visible range. Since our previous study also formulates the conditions of varying the ALD parameters, ${ }^{33}$ we can easily demonstrate that nanostructures, for example nanoneedles, can be grown or deposited for photocatalytic enhancement $^{33}$ or better electron/hole separation. Therefore, within this concept, anisotropic cubic Pd nanoparticles, with different particle sizes $(7,13$ and $22 \mathrm{~nm})$, were properly deposited onto the ZnO@PAN-NF to improve the light absorption capacity of

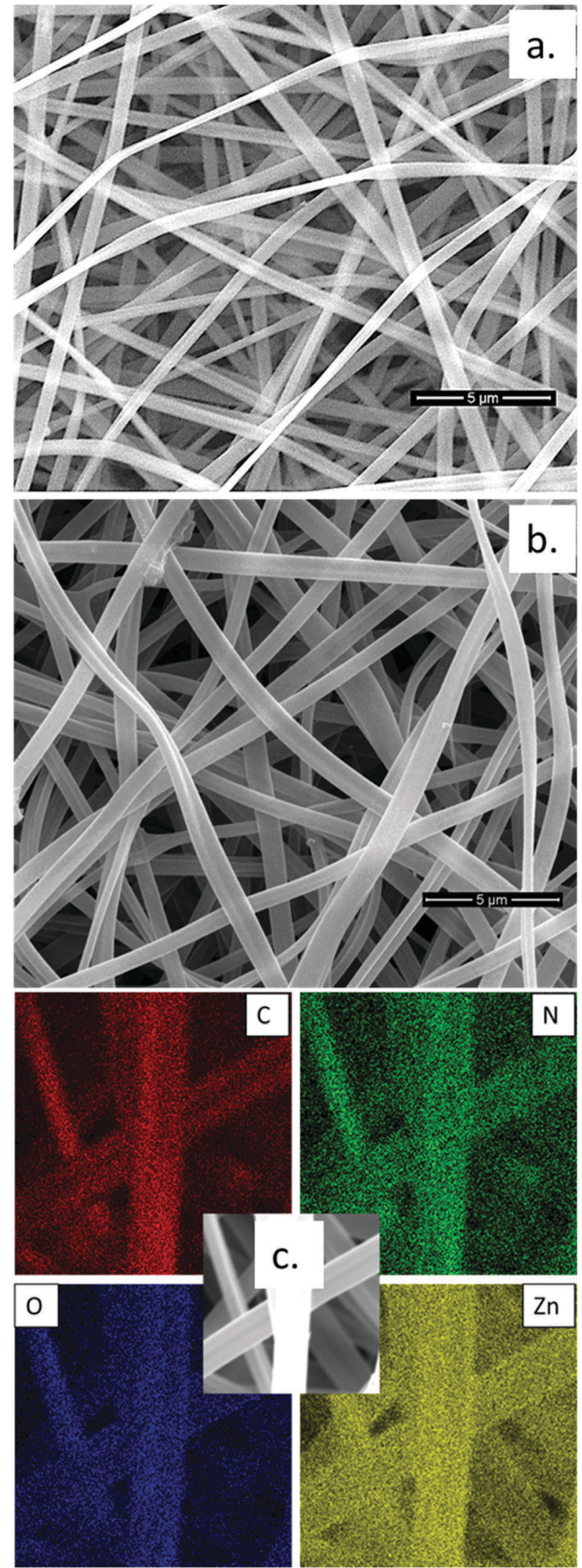

Fig. 2 (a) The representative SEM image of PAN-NF nanofibers, (b) the representative SEM image of ZnO@PAN-NF, and (c) EDX atomic mapping of $\mathrm{ZnO} a \mathrm{PAN}-\mathrm{NF}$. 
this flexible nanofibrous mat of $\mathrm{ZnO@PAN-NF.} \mathrm{Since} \mathrm{the} \mathrm{defects} \mathrm{of}$ $\mathrm{ZnO}$ are capable of coupling with the Pd nanocubes to regulate their plasmonic character, it is promising to use the same structure for an enhanced visible light photocatalytic activity.

For the enhanced plasmonic effect on the ZnO@PAN-NF, cubic Pd nanoparticles with different sizes (7, 13 and $22 \mathrm{~nm}$ ) were synthesized with a thermal decomposition method providing a remarkably fine control of the shape and size (Fig. 3). It is very important to control the intermediate states while forming the Pd nanocubes since chemical affinities play an important role. ${ }^{35-37}$ According to the compositions, it is well known that an acetate precursor of the Pd and additionally I-, Br-, Cl-anions govern the most important aspects of the monodispersed and morphology controlled cube formation. During the nanocube fabrication, when $\mathrm{I}-, \mathrm{Br}-, \mathrm{Cl}-\mathrm{ions}$ are added into the reaction mixture, an intermediate $\left[\mathrm{PdX}_{4}\right]^{2-}$ complex is formed and becomes highly dominant. It is widely known that this complex favorably prefers square planar structures and since PVP molecules can safely stabilize the

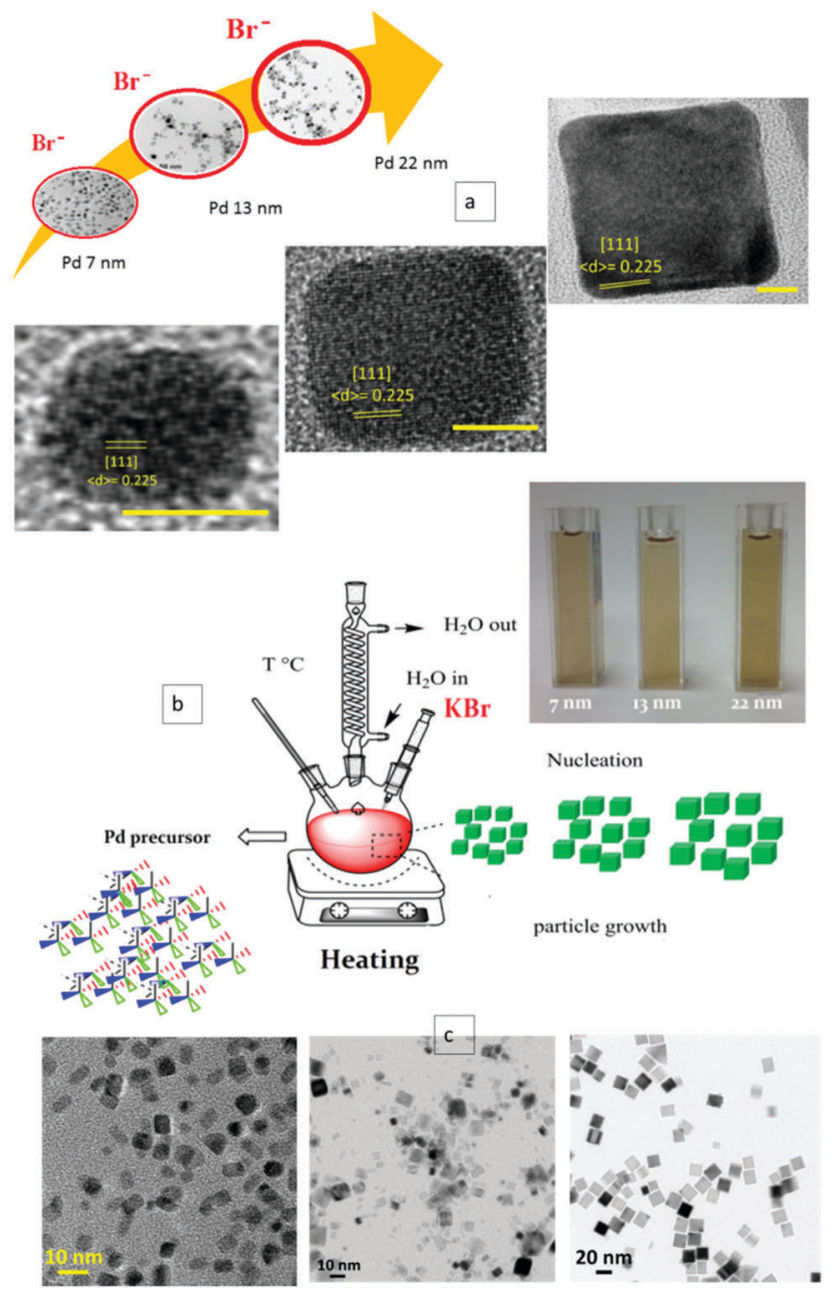

Fig. 3 (a) Br-anion control on the Pd nanocube size and HR-TEM images of a single nanocube (scale bar $=5 \mathrm{~nm}$ ), (b) thermal decomposition method for the Pd precursor and solutions of the obtained Pd nanocubes, and (c) TEM images ( $7 \mathrm{~nm}, 13 \mathrm{~nm}, 22 \mathrm{~nm}$ from left to right, respectively) for the different $\mathrm{Pd}$ nanocubes from a $\mathrm{Na}_{4} \mathrm{PdCl}_{2} \cdot \mathrm{xH}_{2} \mathrm{O}$ precursor.
Br-anions, special facets are preferred and this assistance results in the cube formation. The metallic intermediate $\left[\mathrm{PdX}_{4}\right]^{2-}$ complex to the metal Pd particle procedure follows a reduction path, and there is clear evidences that halogens, in their complex form, easily and selectively undergo reduction to form the nanocubes. ${ }^{38,39}$ Even though the Br-anion was reported in the formation of spherical particles according to the literature, when PVP, temperature, solvent and conditions are finely controlled, anisotropic nanocubes were obtained as presented in this report. PVP favors the formation of nanocubes as a capping polymer of which the oxygen (and/or nitrogen) atoms can bind most strongly to the $\{100\}$ facets of the growing Pd nuclei, causing the formation of nanocubes with a controlled size and truncated corners. Thermodynamically, an fcc single crystal can possibly form from cube to cuboctahedron and then to octahedron with an increasing ratio of [111] to [100] fringe areas. Hence, for the fabrication of single crystalline Pd nanocrystals with facet control, the surface energies of the different facets for growth should be controlled, which indirectly controls the dominant facet formation (Fig. 3a). Synthesized Pd nanocubes were designed with increasing Br-content to modulate their sizes. Therefore, when the Br-anion was used in a lower amount, smaller nanoparticles were obtained $(\sim 7 \mathrm{~nm})$. When the amount of Br-anion was increased 2 fold, $\sim 13 \mathrm{~nm}$ nanocubes were obtained. In the same manner, when the Br-anion was increased 8 fold, $\sim 22 \mathrm{~nm}$ cubic nanoparticles were obtained. HR-TEM results clearly revealed that cubic formation nature of the Pd nanostructures under the presented synthesis conditions did not change, but by increasing the Br-anion concentration we increase the concentration of the intermediate complexes to form and grow the cubic Pd nanoparticles. When the morphology, average particle size and surface characteristics are investigated by HR-TEM (Fig. 3a), all the details showed the mentioned features. In Fig. 3a and c, we present typical TEM images of Pd nanocubes with average sizes of 7, 13 and $22 \mathrm{~nm}$, respectively. In the overview pictures with low-magnification, the TEM images show that Pd nanocubes have well-defined square (cubic) shapes with a narrow size distribution (Fig. S3, ESI $\dagger$ ). In the HR-TEM images of single Pd nanocubes, lattice fringes (Fig. 3a) are clearly visible, of which one can measure an interatomic distance of approximately $0.225 \mathrm{~nm}$ for the [111] facet. Investigation of the HR-TEM image clearly showed that nanocubes have corners with slightly curvy structures but a single-phase structure is clear. The single crystallinity of the Pd nanocubes can also be detected from the appearance of continuous lattice fringes oriented in the same direction across the cube structure of the metal nanoparticle.

TEM (Fig. 4a), HR-TEM (Fig. 4b), XRD (Fig. 4c) and XPS (Fig. 4d) results show the high crystallinity of the $\mathrm{ZnO}$ nanostructure which directly changes the photoluminescence features seen. ${ }^{40,41}$ XRD patterns additionally highlight that if we compare the polar [002] and nonpolar [100] facets of $\mathrm{ZnO}$ layers there is almost a perfect equality $[002] /[100]=1.006$ showing the increased polar character of the $\mathrm{ZnO}$ nanolayer during formation via the ALD process. When the XRD pattern of the ZnO@PAN-NF is examined, it is widely known that pristine PAN may be accompanied with another peak 
in which nanofibers showed a rise at $2 \theta=\sim 17^{\circ}$, corresponding to an orthorhombic (110) reflection. Additionally, in the $2 \theta=20-30^{\circ}$ range another smaller peak corresponding to the (002) reflection can be seen. This is very clearly observable at $2 \theta=\sim 29^{\circ}$ in the XRD diffraction pattern. Starting from $2 \theta=30^{\circ}$, all the peaks recorded correspond to the ZnO, JCPDS: 36-1451 having a hexagonal wurtzite structure. This clearly confirms the successful deposition of $\mathrm{ZnO}$ nanolayers on the electrospun PAN fibers by the ALD technique. When XRD patterns of the Pd decorated ZnO@PAN-NF were compared (Pd@ZnO@PAN-NF), all the peaks corresponding to the hexagonal wurtzite structure of ZnO (JCPDS: 36-1451) showed no variation (Fig. S4, ESI $\dagger$ ). Also, the XRD peak of the PAN structure appears in its usual $\sim 17$ position and does not change its intensity or broadness. Peaks that appeared for Pd@ZnO@PAN-NF at [100], [002] and [101] in the XRD pattern revealed no difference from the non-decorated sample (ZnO@PAN-NF). However, possibly due to the very low concentration and small size, a very weak diffraction line at $40.15^{\circ}$, which corresponds to the metallic $\operatorname{Pd}(111)$ with a face-centered cubic structure, can be seen (Fig. S4, ESI $\dagger$ ). Previous investigations reveal that reflection peaks for Pd nanostructures are generally observed at $40.1^{\circ}, 46.7^{\circ}, 67.8^{\circ}, 82.1^{\circ}$, and $86.4^{\circ}$, which correspond to the [111], [200], [220], [311] and [222] planes of Pd (JCPDS card no. 46-1043), respectively. ${ }^{42,43}$

The crystallinity of $\mathrm{ZnO}$ in the $\mathrm{ZnO} @ \mathrm{PAN}-\mathrm{NF}$ sample is highly visible, which can also be confirmed by the HR-TEM analysis in Fig. 4b. It is well known that the oxygen vacancies present can play a role due to oxygen related crystal stress. Therefore, small variations and different FWHM values can be attributed to the defect oriented crystal formation of the $\mathrm{ZnO}$ nanolayers. The ALD of the ZnO layer onto the PAN nanofibers and the core-shell structure of the ZnO@PAN-NF can also be seen clearly with a uniform $\sim 40 \mathrm{~nm}$ thickness of the ZnO layer (Fig. 4a). Uniformly deposited $\mathrm{ZnO}$ was also seen in the high magnification SEM image (Fig. S5a, ESI $\dagger$ ), in addition, the SAED pattern (Fig. S5b, ESI $\dagger$ ) of ZnO@PAN-NF shows that the $\mathrm{ZnO}$ layer has a polycrystalline nature. The oxygen content from EDX (Fig. S2, ESI $\dagger$ ) and XPS (Fig. 4d) reveals that the ZnO
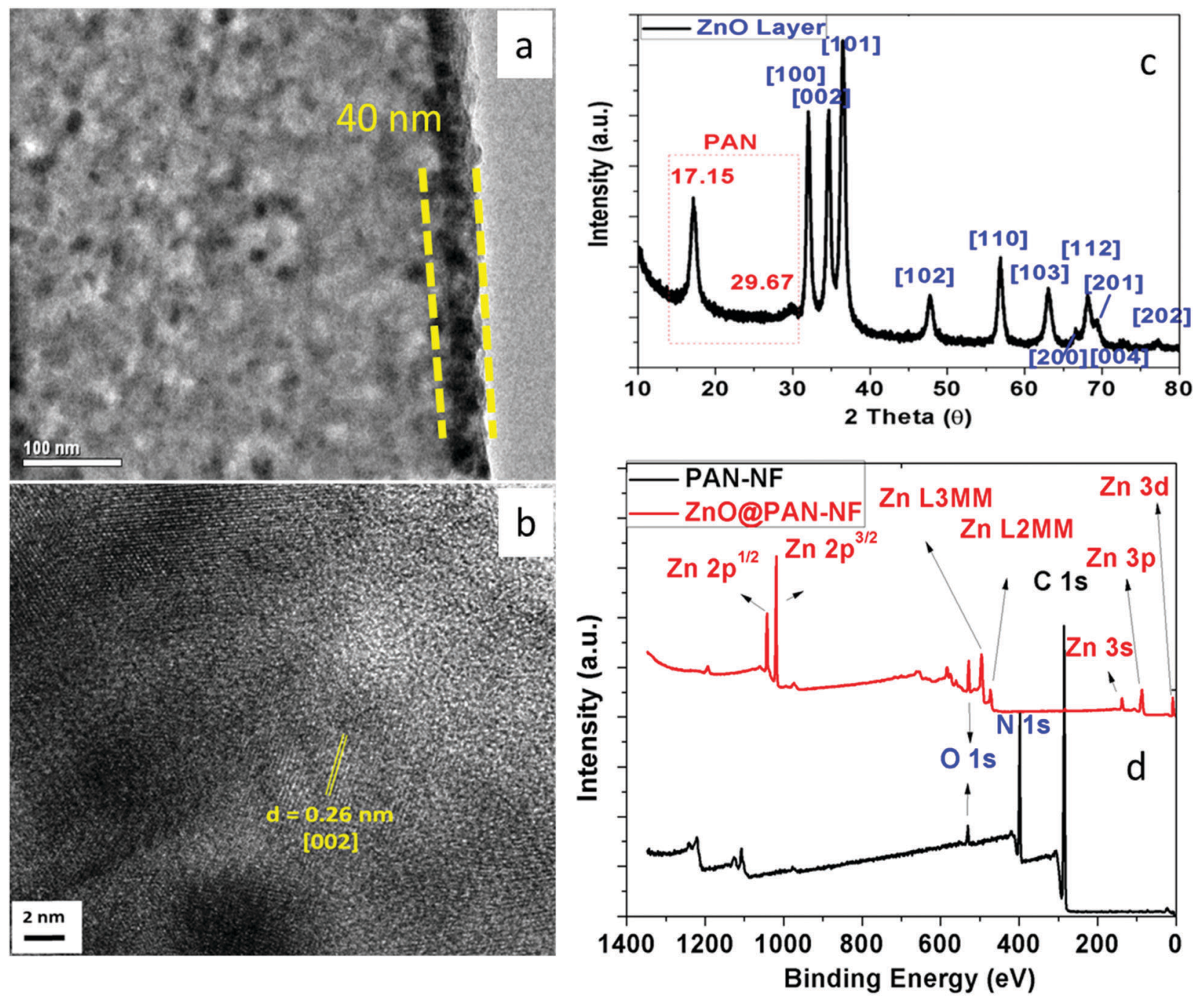

Fig. 4 (a) TEM image of the core-shell nanofibers; ZnO (core)@PAN (shell)-NF, (b) HR-TEM image of ZnO@PAN-NF showing a ZnO layer with [002] facet, (c) XRD pattern of ZnO@PAN-NF, and (d) XPS investigation of the pristine PAN and ZnO coated nanofiber (ZnO@PAN-NF). 
nanolayer is highly oxygen dominant, which provides the defect sites for the photoluminescence feature. In order to enhance the visible range photocatalysis, the $\mathrm{ZnO}$ surface was decorated with Pd nanocubes in which plasmonic transfer can occur. For the nanocube decoration of 3 different $\mathrm{Pd}$ sizes (7, 13 and $22 \mathrm{~nm}$ ), the desired deposition process was performed. Since additional coupling agents, such as mercapto or amino carrying alkoxy silane precursors, would decrease the electron transfer efficiency due to the barrier $\mathrm{SiO}_{2}$ layer formation, direct deposition of the $\mathrm{Pd}$ nanocubes and heat curing was performed. Fig. 5a-c shows the representative TEM and HR-TEM images of the 7, 13 and $22 \mathrm{~nm}$ Pd nanocube decorated $\mathrm{ZnO}$ nanolayers on PAN-NF (Pd@ZnO@PAN-NF). The inner sides of the Pd@ZnO@PAN-NF appear brighter as expected and the edges are darker with $\mathrm{ZnO}$ structures available together with Pd nanocubes. Visual examination clearly showed the Pd nanocube decoration as the pale yellow color of the ZnO@PAN-NF mat transformed into brown-black surfaces (Fig. S6, ESI $\dagger$ ). The $7 \mathrm{~nm}$ nanocubes seem darker visually, but TEM and EDX analyses do not show a remarkable difference about the Pd decoration onto the ZnO@PAN-NF. HR-TEM images of the Pd nanocubes positioned on the $\mathrm{ZnO}$ nanolayers were obtained (Fig. 5a2, b2 and c2). SEM images together with the EDX atomic mapping (Fig. S7, ESI $\dagger$ ) after Pd nanocube decoration for all 3 samples are shown. Additionally, HAADF-STEM-EDS mapping was used to detect the $\mathrm{Pd}, \mathrm{Zn}$ and $\mathrm{O}$ elements in the $\mathrm{Pd}$ nanocube $(7 \mathrm{~nm})$ decorated sample (Pd-7@ZnO@PAN-NF) for a single nanofiber (Fig. 6). Investigation shows that Pd nanocubes were decorated on the Pd@ZnO@PAN-NF, which can enhance the photocatalytic activity in the visible-light range. Different types of exciton emission quenching in semiconductors by different fluorophores, metal nanoparticles or other metal oxide contacts are widely known. ${ }^{44-46}$ Generally, energy transfer occurs from the exciton state and charge carriers in the defect or trap states of nanostructures to the acceptors. During this process the defects are very wide especially in $\mathrm{ZnO}$ nanostructures and due to its natural crystal structure, many origins of the defect states are observable. Quantum yield and catalytic efficiency is also dependent on the defect abundancy and therefore deep investigations are needed for the $\mathrm{ZnO}$ nanostructures. ${ }^{47,48}$ Chemistry related to the visible light emission and relationship between the $\mathrm{ZnO}$ size and emission wavelength is widely studied. It is known that the sources of visible light emission obtained in $\mathrm{ZnO}$ nanostructures can be attributed to the oxygen vacancies, interstitial zinc, chemisorbed oxygen, divalent copper, zinc vacancies and even organic ligands on the surface. Still, we must highlight that there is no fully accepted visible emission mechanism for the $\mathrm{ZnO}$ nanostructures and research results were presented to relate the emission wavelength and chemical structure of the ZnO. Oxygen or air environment
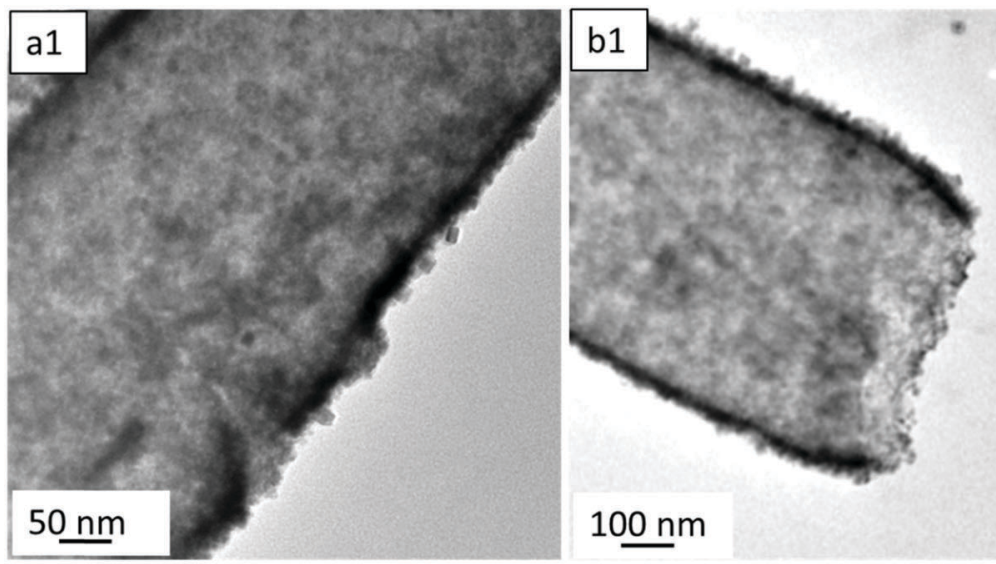

$10 \underline{00 \mathrm{n}}$
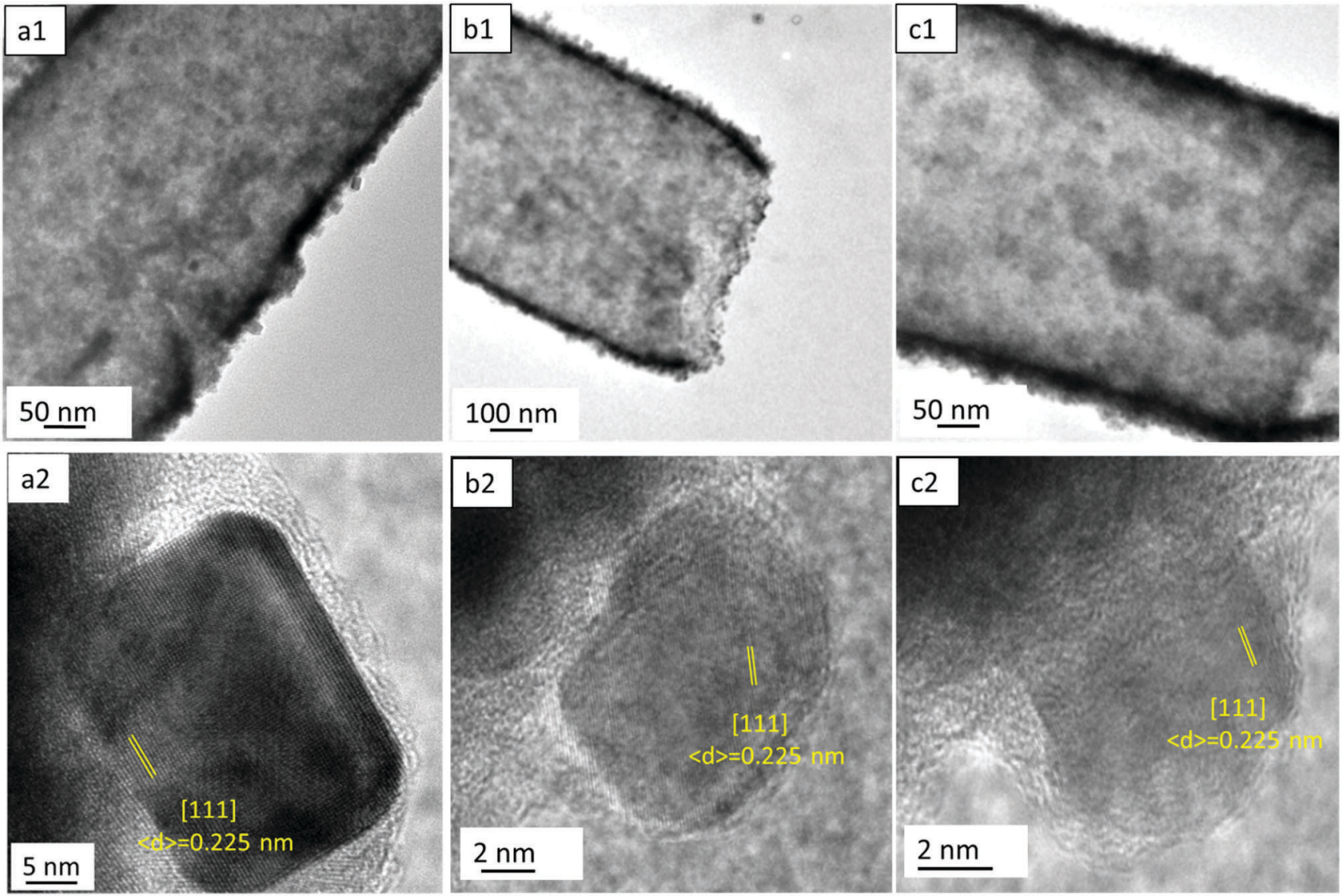

Fig. 5 The representative TEM (1) and HR-TEM (2) images of Pd@ZnO@PAN-NF samples having different size of Pd nanocubes; (a1 and a2) Pd-22@ZnO@PAN-NF, (b1 and b2) Pd-13@ZnO@PAN-NF and (c1 and c2) Pd-7@ZnO@PAN-NF. 

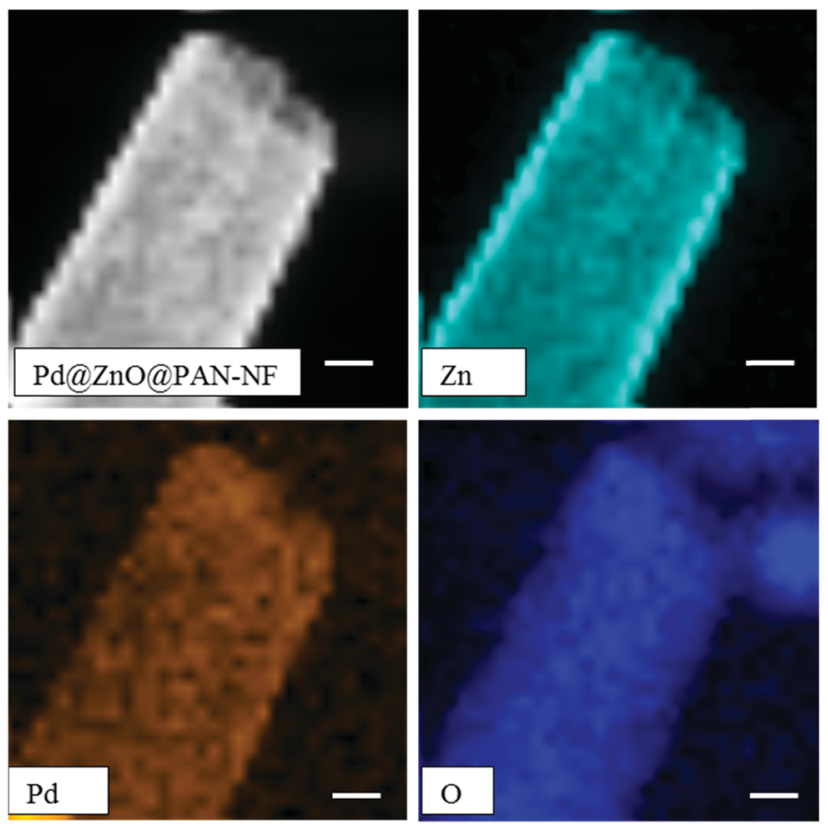

Fig. 6 HAADF-STEM-EDS mapping images obtained from a single nanofiber of Pd-7@ZnO@PAN-NF (scale bar: $200 \mathrm{~nm}$ ).

calcination leads to a reduced visible emission intensity for $\mathrm{ZnO}$ but oxygen vacancies still play a huge role in the $\mathrm{ZnO}$ emission, embarking with the delocalized charge carrier involvement leading a large dipole moment variation causing a possible transfer to the attached fluorophore actors. ${ }^{49,50}$ Accordingly, one can expect the energy transfer from the defect centers as donors to the acceptor structures, but the results are completely different, causing a different interpretation for the conducted experiments. Förster energy transfer theory with dipole moment calculations was utilized to explain the mechanism of the energy transfer from defect states close to the surface, but the donoracceptor distance was small for the covalently bonded fluorophores. Still, contradictory results were observed when there is no covalent bonding, providing a platform to question the FRET mechanism in the present case again. Fig. 7 reveals the measured PL extinction spectra of the ZnO@PAN-NF and Pd@ZnO@PANNF. For comparison of the UV-Vis absorption values, Pd nanocube decorated structures $(7,12$ and $22 \mathrm{~nm})$ were examined and are presented in Fig. S8 (ESI $\dagger$ ) together with the pristine ZnO@PAN-NF. The results revealed an absorption maxima at $\left(\lambda_{\max }=373 \mathrm{~nm}\right)$ for the $\mathrm{ZnO}$ nanolayer on the PAN-NF which is a size related absorption of the band edge character (Fig. S8a, ESI $\dagger$ ). Lower concentrations of the solution did not change the spectral position of the absorption maxima, stressing the remarkable stability of the $\mathrm{ZnO}$ nanolayers. As shown, the ZnO nanolayer did not exhibit any absorption between 400 and $800 \mathrm{~nm}$. However, when Pd was decorated on the ZnO@PAN-NF, an increase in the absorption feature was observed. Since Pd nanocubes have a surface plasmon resonance (SPR) band starting at around $500 \mathrm{~nm}$, this increase can be seen together with a UV region absorption increase. Generally, the surface plasmon resonance (SPR) band for spherical Pd NPs shows a

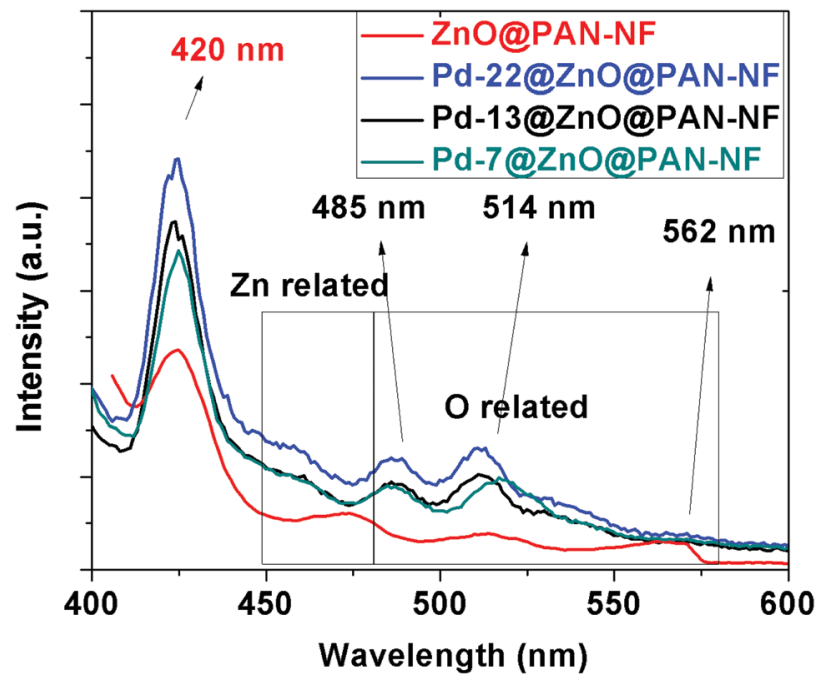

Fig. 7 PL investigation of the ZnO@PAN-NF and PdaZnO@PAN-NF samples.

single band spread over a broad region of $200-700 \mathrm{~nm}$, but when anisotropic shapes are seen, this absorption band splits and shows different maxima depending on the sizes and $\mathrm{D} / \mathrm{L}$ values. For $7 \mathrm{~nm}$ Pd nanocubes, two maxima were observed. The first one was located in the range $250-320 \mathrm{~nm}$ and the second one was located in the $360-490 \mathrm{~nm}$ range. Since PVP is used, the Pd surfaces can also contain small amounts of same molecules but there is no absorption observed at around $212 \mathrm{~nm}$. Accordingly, two maxima were also observed for the 13 and $22 \mathrm{~nm} \mathrm{Pd}$ nanocubes, as presented in Fig. S8b-d (ESI $\dagger$ ). Values change to the $245-280$ and $340-495 \mathrm{~nm}$ ranges for $22 \mathrm{~nm}$ Pd nanocubes and to $260-280 \mathrm{~nm}$ and $370-480 \mathrm{~nm}$ for $13 \mathrm{~nm}$ Pd nanocubes. When the size distribution is obtained, homogeneous decoration and surface area gains importance for the catalytic applications. As prepared Pd nanocubes showed an extreme stability with no sign of oxidation after 5 months, which can be detected via XPS when stored under ambient conditions. Pd nanocube solution colors are already presented in Fig. $3 \mathrm{~b}$. Small variations are observed in the SPR peaks but it was hard to detect a regular increase or decrease concerning the nanocube sizes as reported in the literature. ${ }^{51,52}$

In order to detect the photoluminescence (PL) features of the Pd decorated ZnO nanolayers, we measured the PL characteristics of all three Pd@ZnO@PAN-NFs and compare them with ZnO@PAN-NF, as shown in Fig. 7. As for the ZnO nanolayer, some notable variations are present for the $\mathrm{ZnO}$ defect features. It is known that visible light photocatalysis and emission are surface related properties and once the correct mapping of the $\mathrm{ZnO}$ nanolayer is obtained, their variation with the Pd nanocubes can be defined precisely. The observed PL spectrum of ZnO@PAN-NF consists of a strong UV peak due to near-band-edge emission (NBE) near $395 \mathrm{~nm}$ and a broad visible emissions due to surface and defect related emissions positioned between 450 and $590 \mathrm{~nm}$. When compared, the proportion of the UV emission intensity versus the highest visible emission intensity $\left(I_{\text {uv }} / I_{\text {vis }}\right)$ is around 4 . This is clearly due to the many defect states of the ZnO surface which can be 
classified as $\mathrm{Zn}$ related or O related. Basically, we detected that the PL spectra consist of oxygen vacancies $\left(\mathrm{V}_{\mathrm{O}}\right)$ and zinc interstitials $\left(\mathrm{Zn}_{\text {is }}\right)$ with an almost equal amount of availability observed. It is already known that the formation of the $\mathrm{Zn}_{\text {is }}$ is surface and affinity related when the ALD process is performed. Measured intrinsic defects from the non-equilibrium process related to oxygen are generally more stable and observable when compared to those that originate from zinc and therefore these defect states contribute to the defect related photocatalysis differently. Due to the smaller migration barrier energy of the $\mathrm{Zn}_{\text {is }}$ it is easier to change its chemical nature when compared to $\mathrm{V}_{\mathrm{O}}$. When PL emissions of Pd@ZnO@PAN-NF are investigated, it was noticed that the band gap energy related band appearing at around $420 \mathrm{~nm}$ maintained its position, but the band at $470 \mathrm{~nm}$ red shifted to $485 \mathrm{~nm}$ in all three Pd nanocube decorations. Since these emissions are $\mathrm{Zn}$ based emissions it can be speculated that $\mathrm{Zn}_{\text {is }}$ on the surface was reduced or transformed into different structures after the Pd decoration. Since EDX mapping (Fig. S7, ESI $\dagger$ ) and TEM imaging (Fig. 5) clearly show the availability of the Pd nanocubes, these results can be attributed to the closely attached Pd nanocube decoration. Since nanocube size relates to large surface areas, the attachment and positioning on the $\mathrm{ZnO}$ layer may change the surface of the $\mathrm{ZnO}$ nanolayer and provide the defect oriented electron and charge transfer, as evidenced by PL results. During the Pd nanocube decoration and heat treatment, $\mathrm{Zn}$ species were allowed to obtain enough energy for the migrational movement and the related PL emission decreased or vanished. On the other hand, oxygen related emission at $514 \mathrm{~nm}$ and $562 \mathrm{~nm}$ seems relatively decreased after the Pd nanocube deposition. The PL spectrum of the all-Pd nanocube decorated ZnO@PAN-NF shows that when the size of the nanocube gets smaller, the intensity of the UV peak is also reduced. However, when measured after the Pd nanocube decoration, the intensity ratio of the UV emission to the highest visible emission $(514 \mathrm{~nm})$ slightly reduced to an average 2.2, which is less than that for pristine ZnO@PAN-NF. Specifically, if the PL spectra are evaluated individually, $7 \mathrm{~nm}$ Pd nanocube decorated ZnO@PAN-NF shows the lowest emission values as seen in Fig. 7. As the sizes of the Pd nanocubes are increased to $13 \mathrm{~nm}$ and $22 \mathrm{~nm}$, the intensities are relatively higher. These findings can be explained by the photoelectron transfer triggered by the Pd nanocube decoration.

Fig. 8a shows defect oriented and plasmon enhanced photocatalytic activity of the Pd@ZnO@PAN-NF studied by methylene blue $(\mathrm{MB})$ dye degradation. When photoexcited, recombination between the holes in $\mathrm{ZnO} \mathrm{VB}$ and electrons in a deep defect level can induce the visible-range emission, which is generally known as nonradiative recombination. It is clear that after Pd nanocube decoration, visible light emission and visible light photocatalytic activity increased and the underlying mechanism can be suggested as follows. Since the SPR energy of the Pd nanocubes is close to the defect emission of the ZnO nanolayer on the PAN-NF, the energy of the visible light emission can be absorbed by these Pd nanocubes during the excitation. In addition to that, resonant excitation for the Pd nanocubes fabricates the highly energetic electrons which can be transferred back into the $\mathrm{CB}$ of the $\mathrm{ZnO}$
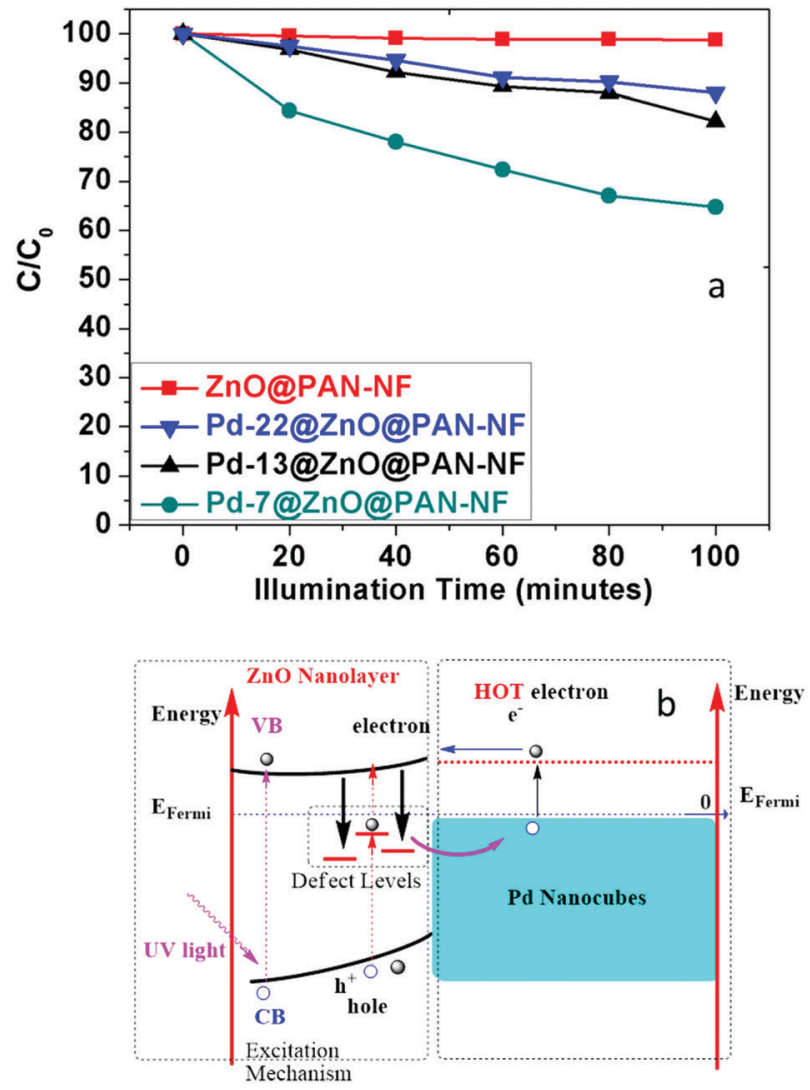

Fig. 8 (a) Photocatalytic degradation of MB dye by PdaZnO@PAN-NF samples under visible light illumination, and (b) defect enhanced plasmonic electron transfer for visible light photocatalysis mechanism for Pd@ZnO@PAN-NF.

nanolayer. ${ }^{53,54}$ This phenomenon unveils the energy emission of the $\mathrm{ZnO}$ nanolayer. It is clear that the excited match between the SP absorption of the Pd nanocubes and the defect surface plasmons of the Pd nanocubes can be transferred into the CB of $\mathrm{ZnO}$ together with some recombined electrons and holes. Energetic electrons can relax very fast and combination with the holes in the VB on the $\mathrm{ZnO}$ nanolayer provides an increase in intensity. In addition to that, when the Pd nanocube decoration is employed well, the transfer of the electrons can result in an increase in the band emission as seen in Fig. 8b. The depicted scheme of the underlying mechanism is shown together with the photocatalytic comparison of the Pd decorated ZnO@PAN-NF. According to the scheme, an energy transfer from the ZnO layer to the Pd nanocubes and electron transfers from these nanocubes to the VB of the ZnO is seen. By the whole system analyzed here, we can simply guess that these visible excitations originated from defect states for the hot electrons that could trigger the visible range photocatalytic activity of the Pd@ZnO@PAN-NF system. Therefore, catalytic degradation of the MB dye was selected as a proof-of-concept study (Fig. S9, ESI $\dagger$ ). The decomposition rate of $\mathrm{MB}$ degradation was quantified via a first order exponential fit for the obtained aliquots and data set

$$
\ln C_{0} / C=k t
$$


where $C_{0}$ and $C$ are the concentrations of $\mathrm{MB}$ at different irradiation times at the beginning $\left(t_{0}\right)$ and $t$, respectively. Additionally, $k$ is the pseudo-first-order rate constant of photodegradation $\left(\min ^{-1}\right)$. From the linear fitting curves of $\ln \left(C_{0} / C\right)$ versus irradiation time $t$, the $\mathrm{MB}$ degradation rate constant $k$ can be calculated. Kinetics of the degradation for each sample (Fig. S9, ESI $\dagger$ ) showed that the $7 \mathrm{~nm}$ Pd nanocube decorated sample showed the highest photocatalytic activity (Fig. 8a). We can easily attribute these increases in photocatalytic activity to the Pd decoration since ZnO@PAN-NF did not show any decomposition of the MB dye (Fig. 8a and Fig. S9, ESI $\dagger$ ).

Since the attachment of the Pd nanocubes changes the nature of the charge separation process, it was concluded that Pd nanocubes and their electronic band structure together with SPR contributed to the photocatalytic activity. It is known that upon illumination, electron-hole pairs are formed and they are separated if the proper conditions are provided. Since the fundamental aim is to find electron acceptors, such as $\mathrm{O}_{2}$, to form superoxide radicals $\left({ }^{\bullet} \mathrm{O}^{2-}\right)$ and hole acceptors, such as $-\mathrm{OH}$, to form $-\mathrm{OH}^{\bullet}$ hydroxyl radicals, all the necessary conditions are available after illumination. ${ }^{55,56}$ Still, it is known that these formed radicals can be quenched or recombined by different sources and cannot work as oxidants or reducing agents. When Pd nanocubes were decorated onto the ZnO@PN-NF, certain observations were made. Firstly, since Pd nanocubes act as the removing agent for the electrons formed by illumination, recombination with the holes is prevented. Secondly, since recombination decreases, an increase in photocatalysis is clear. But in our experiment, in addition to these clear expectations, defect levels positioned in between the $\mathrm{VB}$ and $\mathrm{CB}$ of the ZnO@PAN-NF structures can trap the electrons and transfer them to the Pd nanocubes due to the low energy barrier. This basically does not need a UV level energy and can work with visible light energy. According to the results, when the $\mathrm{Pd}$ nanocube size increases, visible light photocatalytic activity decreases, which can be explained by the higher surface area of the smaller nanocubes and relatively higher deposited concentration of the Pd-7 nanocubes. The low concentration of the Pd-22 may cause weakly attached Pd nanocubes to decrease the defect-level electron transfer to the Pd nanocubes. Since bigger Pd nanocubes can be positioned with different edges and restrict a better contact with the $\mathrm{ZnO}$ surfaces causing relatively smaller transfer, low photocatalytic activity was observed. Smaller Pd nanocubes provide better contacts or increased heterojunctions, which means a higher activity. The results clearly convinced us that size controlled anisotropic Pd nanoparticles can enhance the visible range photocatalytic activity of the ZnO@PAN-NF. The ALD method provided an extreme flexibility for the highly crystalline $\mathrm{ZnO}$ nanolayers deposited onto the PAN nanofibers fabricated by the electrospinning method.

From the literature, we know that the electrospun nanofibrous mats have $\sim 10-20 \mathrm{~g} \mathrm{~m}^{-2}$ specific surface area in general, ${ }^{57,58}$ but the visible light photocatalysis is directly related with the Pd nanocube decoration in this study. Therefore, for the analysis of the Pd nanocube decoration effect, a representative high resolution XPS analysis of the Pd $3 \mathrm{~d}$ and $\mathrm{Zn} 2 \mathrm{p}$ are presented for each Pd@ZnO@PAN-NF samples (Fig. S10, ESI $\dagger$ ). According to the XPS data, all the anticipated atomic entities are available, as expected. $\mathrm{Zn}$ peaks appear as $\mathrm{Zn} 2 \mathrm{p}_{3 / 2}$ and $\mathrm{Zn} 2 \mathrm{p}_{1 / 2}$ subpeaks of the $\mathrm{Zn} 2 \mathrm{p}$ doublet, which are located at 1020.85 and $1044.2 \mathrm{eV}$, respectively. For the Pd nanocubes, generally double peaks with the binding energies of 334.7-334.9 and 339.8-340.0 eV corresponding to $\mathrm{Pd} 3 \mathrm{~d}_{5 / 2}$ and $\mathrm{Pd} 3 \mathrm{~d}_{3 / 2}$, respectively, were observed. Additionally, oxidized PdO peaks were also detected in the 337.2-337.5 and 342.3-342.5 eV range, respectively. The latter XPS values are typical valence state peaks of PdO and are found to be consistent with the literature values. ${ }^{59}$ The $13 \mathrm{~nm} \mathrm{Pd}$ decorated structure shows relatively low intensities, but still Pd 3d peak positions are visible. According to the compositional analysis, Pd-7 decorated ZnO@PAN-NF hosts roughly 30\% more Pd nanocubes when compared to the Pd-13 and Pd-22 nm decorated samples. To support this information, we have also conducted ICP-MS analyses for the Pd@ZnO@PAN-NF samples (Fig. S11, ESI $\dagger$ ). According to the ICP-MS results, Pd-7@ZnO@ PAN-NF showed the highest Pd concentration when compared to the Pd-13@ZnO@PAN-NF and Pd-22@ZnO@PAN-NF samples. ICP-MS results revealed that the Pd-7@ZnO@PAN-NF sample contains almost 45-50\% more Pd nanocubes than the Pd-13@ ZnO@PAN-NF sample. Additionally, the Pd-22@ZnO@PAN-NF sample shows the lowest Pd content. According to XPS (Fig. S10, $\mathrm{ESI} \dagger$ ) and ICP-MS (Fig. S11, ESI $\dagger$ ) analyses, the Pd content varied as Pd-7@ZnO@PAN-NF > Pd-13@ZnO@PAN-NF > Pd-22@ ZnO@PAN-NF. These findings also provide a close understanding of the Pd nanocube decoration since a relatively higher content and better attachment for the $\mathrm{Pd}-7$ nanocubes enhance the efficiency of the Pd-7@ZnO@PAN-NF for visible light photocatalysis.

In the literature, ${ }^{60}$ a similar plasmon enhanced mechanism with Ag@ZnO flowers improved the visible light photocatalytic activity with a back feeding mechanism. In another study, even though the size of the Pd was not effectively investigated, alkene reactions were shown to be highly improved. ${ }^{61}$ An interesting review listing previous mono and bi-structured plasmonenhanced studies can also give a deep insight on the catalytic applications. $^{62}$ Results showed that the plasmon enhanced methods were applied with an engineered mechanism but the present Pd decoration resulted in a highly noticeable improvement. Especially, the size dependent decoration effects and optical features represent a new insight for light triggered catalytic applications.

It is worth mentioning that we have a remarkable control of every fabrication step of the core-shell ZnO@PAN-NF structure to design this enhanced electron/hole separation system. ALD has huge advantages to deposit highly crystalline, thickness and defect controlled $\mathrm{ZnO}$ nanolayers which have the main role for the photocatalytic activity. Deposition cycle numbers, nanolayer thicknesses and crystallinity are under the control of the ALD process. By combining these well-controlled nanofabrication techniques, we have synthesized anisotropic Pd nanoparticles of different sizes and decorated onto the $\mathrm{ZnO}$ nanolayers of the core-shell ZnO@PAN-NF to enhance the 
plasmonic transfer for visible light photocatalytic activity. Since the contact features and surface area of the $\mathrm{ZnO}$ nanolayers are better for smaller cubic Pd nanoparticles, one can clearly expect an enhanced plasmonic transfer and visible range photocatalytic character as supported by the presented results, which is a novel way to produce plasmon enhanced visible photocatalytic active nanomaterials.

\section{Conclusions}

Here, we have described the fabrication of a flexible nanofibrous composite mat based on Pd nanocube decorated core-shell nanofibers of a polymer (PAN) as the core and a metal oxide (ZnO) as the shell (ZnO@PAN-NF) obtained by electrospinning and ALD nanofabrication methods. The obtained Pd@ZnO@ PAN-NF mats provided an enhanced visible light photocatalytic activity. After the synthesis of Pd nanocubes in three different size $(7,13$ and $22 \mathrm{~nm})$, surface plasmon mediated enhancement was acquired on the ZnO@PAN-NF by the Pd nanocube decoration. Due to the suitable $\mathrm{ZnO}$ surface, higher surface areas with different Pd nanocube sizes, defect energies and good contact architecture were obtained. The results showed that between the Pd nanocubes and $\mathrm{ZnO}$ nanolayer, energy transfer was possible. Additionally, plasmonic resonance was excited by the defect electrons of the $\mathrm{ZnO}$ nanolayer providing increased visible light photocatalytic activity. The electron-hole recombination rate of the photogenerated electrons and holes was reduced by the electron trapping via Pd nanocubes. This simply provides the enhanced photocatalytic activity, as an analytical investigation also supported the MB dye degradation in aqueous medium.

\section{Acknowledgements}

Dr Arslan acknowledges The Scientific \& Technological Research Council of Turkey (TUBITAK), TUBITAK BIDEB Fellowships for 2232 programme (project no. 115C095) and financial support. T. U. acknowledges partial support of The Turkish Academy of Sciences-Outstanding Young Scientists Award Program (TUBA-GEBIP). The authors thank Mustafa Güler for TEMSTEM imaging. We also acknowledge the help of Nuray Gündüz for ICP-MS measurements.

\section{Notes and references}

1 S. Banerjee, S. C. Pillai, P. Falaras, K. E. O'Shea, J. A. Byrne and D. D. Dionysiou, J. Phys. Chem. Lett., 2014, 15, 2543.

2 H. Tong, S. Ouyang, Y. Bi, N. Umezawa, M. Oshikiri and J. Ye, Adv. Mater., 2012, 24, 229.

3 H. Kisch, Angew. Chem., Int. Ed., 2013, 52, 812.

4 M. G. Méndez-Medrano, E. Kowalska, A. Lehoux, A. Herissan, B. Ohtani, S. Rau, C. Colbeau-Justin, J. L. RodríguezLópezand and H. Remita, J. Phys. Chem. C, 2016, 43, 25010.
5 I. Vukoje, T. Kovač, J. Džunuzović, E. Džunuzović, D. Lončarević, S. P. Ahrenkiel and J. M. Nedeljković, J. Phys. Chem. C, 2016, 33, 18560.

6 R. Asahi, T. Morikawa, T. Ohwaki, K. Aoki and Y. Taga, Science, 2001, 293, 269.

7 S. Bingham and W. A. Daoud, J. Mater. Chem., 2011, 21, 2041-2050.

8 K. S. Ranjith, A. Senthamizhan, B. Balusamy and T. Uyar, Catal. Sci. Technol., 2017, 7, 1167.

9 Z. Wang, Y. Liu, B. Huang, Y. Dai, Z. Lou, G. Wang, X. Zhang and X. Qin, Phys. Chem. Chem. Phys., 2014, 16, 2758.

10 H. Cheng, K. Fuku, Y. Kuwahara, K. Moriab and H. Yamashita, J. Mater. Chem. A, 2015, 3, 5244.

11 S. N. Habisreutinger, L. Schmidt-Mende and J. K. Stolarczyk, Angew. Chem., Int. Ed., 2013, 52, 7372.

12 C. Karunakaran and P. Vinayagamoorthy, New J. Chem., 2016, 40, 1845.

13 J. Low, J. Yu, Q. Li and B. Cheng, Phys. Chem. Chem. Phys., 2014, 16, 1111.

14 S. G. Kumar and K. S. R. K. Rao, Appl. Surf. Sci., 2017, 391, 124.

15 W. Yu, J. Zhang and T. Peng, Appl. Catal., B, 2016, 181, 220.

16 V. E. Podasca, T. Buruiana and E. C. Buruiana, Appl. Surf. Sci., 2016, 377, 262.

17 T. Parandhaman, N. Pentela, B. Ramalingam, D. Samanta and S. K. Das, ACS Sustainable Chem. Eng., 2017, 5, 1033-1045.

18 S. Girish Kumar and K. S. R. Koteswara Rao, RSC Adv., 2015, $5,3306$.

19 Z. A. Lewicka, W. W. Yu, B. L. Oliva, E. Quevedo Contreras and V. L. Colvin, J. Photochem. Photobiol., A, 2013, 263, 24.

20 A. Sirelkhatim, S. Mahmud, A. Seeni, N. Haida, M. Kaus, L. C. Ann, S. Khadijah, M. Bakhori, H. Hasan and D. Mohamad, Nano-Micro Lett., 2015, 7, 219.

21 J. Huang, Z. Yin and Q. Zheng, Energy Environ. Sci., 2011, 4, 3861-3877.

22 J. Xu, Z. Chen, J. Antonio Zapien, C. Lee and W. Zhang, Adv. Mater., 2014, 26, 5337.

23 F. Kayaci, C. Ozgit-Akgun, N. Biyikli and T. Uyar, RSC Adv., 2013, 19, 6817.

24 E. Della, G. Anthony, S. R. Chesman, J. Embdenand and J. J. Jasieniak, ACS Nano, 2014, 9, 9154.

25 T. D. Dao, G. Han, N. Arai, T. Nabatame, Y. Wada, C. V. Hoang, M. Aono and T. Nagao, Phys. Chem. Chem. Phys., 2015, 17, 7395.

26 Z. Yi, J. Chen, J. Luo, Y. Yi, X. Kang, X. Ye, P. Bi, X. Gao, Y. Yi and Y. Tang, Plasmonics, 2015, 10, 1373.

27 W. He, H. Wu, W. G. Wamer, H. Kim, J. Zheng, H. Jia, Z. Zheng and J. Yin, ACS Appl. Mater. Interfaces, 2014, 17, 15527.

28 P. Huang, D. H. Kim and J. Lee, Appl. Phys. Lett., 2014, 104, 142102.

29 M. Farbod, M. K. Rasool and M. D. Talebzadeh, Plasmonics, 2016, DOI: 10.1007/s11468-016-0323-1.

30 J. H. Wendorff, S. Agarwal and A. Greiner, Electrospinning: materials, processing, applications, John Wiley \& Sons, Weinheim, 2012. 
31 O. Arslan, Z. Aytac and T. Uyar, ACS Appl. Mater. Interfaces, 2016, 8, 19747.

32 O. Arslan, Z. Aytaç and T. Uyar, J. Mater. Chem. C, 2017, 5, 1816.

33 F. Kayaci, S. Vempati, C. Ozgit-Akgun, N. Biyikli and T. Uyar, Appl. Catal., B, 2014, 156-157, 173.

34 A. E. Deniz, A. Celebioglu, F. Kayaci and T. Uyar, Mater. Chem. Phys., 2011, 129, 701.

35 T. Teranishi and M. Miyake, Chem. Mater., 1998, 10, 594.

36 B. Lim, M. Jiang, J. Tao, P. H. C. Camargo, Y. Zhu and Y. Xia, Adv. Funct. Mater., 2009, 19, 189.

37 J. Mondal, R. Singuru, K. Dhanalaxmi, S. C. Shit and B. M. Reddy, ChemCatChem, 2017, DOI: 10.1002/cctc.201700186.

38 I. Saldan, Y. Semenyuk, I. Marchuk and O. Reshetnyak, J. Mater. Sci., 2015, 50, 2337.

39 C. J. Murphy, T. K. Sau, A. M. Gole, C. J. Orendorff, J. Gao, L. Gou, S. E. Hunyadi and T. Li, J. Phys. Chem. B, 2005, 109, 13857.

40 O. Arslan, L. Belkoura and S. Mathur, J. Mater. Chem. C, 2015, 3, 11965.

41 O. Arslan, A. P. Singh, L. Belkoura and S. Mathur, J. Mater. Res., 2013, 28, 1947.

42 S. Yang, C. Shen, Y. Tian, X. Zhang and H. Gao, Nanoscale, 2014, 6, 13154-13162.

43 R. Wojcieszak, M. J. Genet, P. Eloy, P. Ruiz and E. M. Gaigneaux, J. Phys. Chem. C, 2010, 114, 16677-16684.

44 J. Shi, F. Tian, J. Lyu and M. Yang, J. Mater. Chem. B, 2015, 3, 6989.

45 FRET-Förster Resonance Energy Transfer, From Theory to Applications, 2014 Wiley-VCH Verlag GmbH \& Co. KGaA, Dr Igor Medintz, Dr Niko Hildebrandt, 2014.

46 J. Y. Chang, T. G. Kim and Y. M. Sung, Nanotechnology, 2011, 42, 425708.
47 I. L. Medintz, A. R. Clapp, H. Mattoussi, E. R. Goldman, B. Fisher and J. M. Mauro, Nat. Mater., 2003, 2, 630.

48 J. Wang, Z. Wang, B. Huang, Y. Ma, Y. Liu, X. Qin, X. Zhang and Y. Dai, ACS Appl. Mater. Interfaces, 2012, 4, 4024.

49 Y. Zheng, C. Chen, Y. Zhan, X. Lin, Q. Zheng, K. Wei, J. Zhu and Y. Zhu, Inorg. Chem., 2007, 46, 6675.

50 S. M. Ng, M. Koneswaran and R. Narayanaswamy, RSC Adv., 2016, 6, 21624.

51 J. Villanueva-CabJose, L. Montaño-Priedeand and U. Pal, J. Phys. Chem. C, 2016, 19, 10129.

52 E. L. Runnerstrom, A. Bergerud, A. Agrawal, R. W. Johns, C. J. Dahlman, A. Singh, S. M. Selbachand and D. J. Milliron, Nano Lett., 2016, 5, 3390.

53 F. Kayaci, S. Vempati, I. Donmez, N. Biyikli and T. Uyar, Nanoscale, 2014, 6, 10224.

54 F. Kayaci, S. Vempati, C. Ozgit, I. Donmez, N. Biyikli and T. Uyar, Nanoscale, 2014, 6, 5735.

55 M. Hayyan, M. A. Hashimand and I. M. Al Nashef, Chem. Rev., 2016, 5, 3029.

56 W. Hou and S. B. Cronin, Adv. Funct. Mater., 2013, 23, 1612.

57 F. Kayaci, Z. Aytac and T. Uyar, J. Hazard. Mater., 2013, 261, 286.

58 A. Senthamizhan, B. Balusamy, Z. Aytac and T. Uyar, CrystEngComm, 2016, 18, 6341.

59 A. Jürgensen, N. Heutz, H. Raschke, K. Merz and R. Hergenröder, Anal. Chem., 2015, 87, 7848.

60 Y. Liang, N. Guo, L. Li, R. Li, G. Ji and S. Gan, New J. Chem., 2016, 40, 1587.

61 J. Mondal, Q. T. Trinh, A. Jana, W. K. H. Ng, P. Borah, H. Hirao and Y. Zhao, ACS Appl. Mater. Interfaces, 2016, 8, 15307.

62 X. Zhou, G. Liu, J. Yu and W. Fanc, J. Mater. Chem., 2012, 22, 21337. 